\title{
AUTOMORPHISM GROUPS OF LAMINATED NEAR-RINGS
}

\author{
by K. D. MAGILL, JR. \\ (Received 19th September 1978)
}

\section{Introduction}

Let $N$ be an arbitrary near-ring. Each element $a \in N$ determines in a natural way a new multiplication on the elements of $N$ which results in a near-ring $N_{a}$ whose additive group coincides with that of $N$ but whose multiplicative semigroup generally differs. Specifically, we define the product $x * y$ of two elements in $N_{a}$ by $x * y=x a y$ where a product in the original near-ring is denoted by juxtaposition. One easily checks that $N_{a}$ is a near-ring with addition identical to that of $N$. The original near-ring $N$ will be referred to as the base near-ring, $N_{a}$ will be referred to as a laminated near-ring of $N$ and $a$ will be referred to as the laminating element or sometimes more simply as the laminator.

Throughout this paper $N$ will be the near-ring of all continuous selfmaps of the real numbers under pointwise addition and composition of functions. For our laminating element, we take any odd degree polynomial $P$ and we completely determine the automorphism group of the laminated near-ring $N_{P}$. We will find that there are precisely three possibilities. Aut $N_{P}$ is isomorphic to either the multiplicative group of nonzero real numbers, the cyclic group of order two or the trivial one element group. Furthermore, it will be apparent that in most instances, $N_{P}$ is rigid. That is, it is the third possibility which generally prevails.

A slight digression is appropriate at this point. The multiplicative part of the near-ring is, of course, a semigroup and there is the problem of determining its automorphism group. The solution to that problem turns out to differ considerably from the analogous problem for near-rings which we treat in this paper. The automorphism group of the laminated semigroup turns out to be isomorphic to a Schützenberger group of the base semigroup. However, this is not really the place to go into the details and we leave that for a future paper.

\section{The main results}

Let us first agree upon some notation. The product of two elements $f$ and $g$ in $N$ is their composition and will be denoted by $f \circ g$. The product of those same two elements in the laminated near-ring $N_{P}$ will be denoted by $f g$. In other words $f g=f \circ P \circ g$. And now we proceed with 
Theorem 2.1. Let $P$ be any odd degree polynomial. Then the following statements are equivalent

$N_{P}$ has more than two automorphisms;

Aut $N_{P}$ is isomorphic to $R_{M}$ the multiplicative group of nonzero real numbers;

$N_{P}$ is isomorphic to $N$;

$P$ is a homeomorphism.

Proof. We show first that (2.1.1) implies (2.1.4). Let $\varphi$ be an automorphism of $N_{P}$. According to Theorem (3.1) of $(2$, p. 286) there exists a homeomorphism $h$ from $R$ onto $R$ and a topological isomorphism $t$ from the additive group $R$ of real numbers such that for each $f$ in $N_{P}$ the following diagram commutes

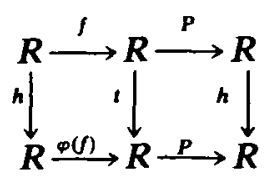

We next wish to note that if $\varphi_{1}$ and $\varphi_{2}$ are two different automorphisms then the two topological isomorphisms $t_{1}$ and $t_{2}$ associated with them as in diagram (2.1.5) must also be different. Suppose to the contrary, that $t_{1}=t_{2}$ and let $h_{i}$ be the two homeomorphisms associated with $\varphi_{i}(i=1,2)$ as in (2.1.5). Then because the diagram commutes, we have

$$
h_{1} \circ P=P \circ t_{1}=P \circ t_{2}=h_{2} \circ P
$$

Since $P$ is surjective, this means that $h_{1}=h_{2}$ and this fact together with $t_{1}=t_{2}$ implies $\varphi_{1}=\varphi_{2}$ in view of diagram (2.1.5).

Now let us return to the automorphism $\varphi$ and the associated topological isomorphism $t$. There exists a nonzero real number $a$ such that $t(x)=a x$ for each $x \in R$. Since $N_{P}$ has at least three automorphisms and since different automorphisms give rise to different topological isomorphisms, we are justified in assuming that $a \neq 1,-1$. We can, in fact, assume still more without any loss in generality. We can assume $a>1$. The reasons for this follow. If $a<0$, choose the automorphism $\varphi^{2}$. One verifies that the topological isomorphism associated with $\varphi^{2}$ is $t^{2}$ which means $t^{2}(x)=a^{2} x$ for all $x$. If $a^{2}>1$ we are through. If not, choose the automorphism $\left(\varphi^{2}\right)^{-1}=\varphi^{-2}$. One then verifies that the topological isomorphism associated with $\varphi^{-2}$ is $t^{-2}$ which is given by $t^{-2}(x)=a^{-2} x$ and since $0<a^{2}<1$ we have $a^{-2}>1$. Thus, we may, as we previously asserted, assume that $a>1$.

Now we consider the automorphism $\varphi^{n}$, that is, the $n$th iteration of $\varphi$. The homeomorphism associated with $\varphi^{n}$ is $h^{n}$ and the topological isomorphism is $t^{n}$ where $h$ and $t$ are the maps associated with $\varphi$. Thus, $t^{n}(x)=a^{n} x$ for each $x \in R$. Suppose $P(x)=P(y)$. Because $\varphi^{n}, h^{n}$ and $t^{n}$ satisfy a commutative diagram like (2.1.5), we have

$$
P\left(a^{n} x\right)=P \circ t^{n}(x)=h^{n} \circ P(x)=h^{n} \circ P(y)=P \circ t^{n}(y)=P\left(a^{n} y\right)
$$

and this holds for each positive integer $n$. We want to show that $x=y$. If $x=y=0$ we 
are through. Otherwise, one of them, say $y \neq 0$. Since $P$ is an odd degree polynomial and since $a>1$, we can choose $n$ so large that

$$
P^{-1}\left(P\left(a^{n} y\right)\right)=\left\{a^{n} y\right\} .
$$

This, together with (2.1.6) implies that $a^{n} x=a^{n} y$ which, in turn, implies that $x=y$. Thus, $P$ is injective and this completes the verification that (2.1.1) implies (2.1.4).

Now assume that (2.1.4) holds and define a map from $N_{P}$ into $N$ by $\varphi(f)=f \circ P$. Continuity of $P$ alone is sufficient to insure that $\varphi$ is a homomorphism. Furthermore, $\varphi$ is injective because $P$ is surjective and $\varphi$ is surjective because $P$ is a homeomorphism. Consequently, (2.1.4) implies (2.1.3).

We established in (1) (see Corollary 4.3 and Example 4.4, p. 105) that Aut $N$ is isomorphic to $R_{M}$ so that (2.1.3) implies (2.1.2) and since (2.1.2) so obviously implies (2.1.1) the proof of the theorem is complete.

Now we consider the case where $N_{P}$ has exactly two automorphisms and this compels us to introduce the following

Definition 2.2. A continuous function $f$ mapping $R$ into $R$ is pseudosymmetric if $f(a)=f(b)$ implies $f(-a)=f(-b)$.

Theorem 2.3. The automorphism group Aut $N_{P}$ of the laminated near-ring $N_{P}$ is isomorphic to $Z_{2}$ the cyclic group of order two if and only if $P$ is pseudosymmetric and not a homeomorphism.

Proof. Suppose first that $P$ is pseudosymmetric and not a homeomorphism. In view of Theorem 2.1 Aut $N_{P}$ cannot have more than two elements so we need only show that it has an automorphism other than the identity. With this in mind, we define a mapping $h$ from $R$ into $R$ as follows: let any $y \in R$ be given. Choose any $x \in R$ such that $P(x)=y$ and define

$$
h(y)=P(-x) \text {. }
$$

Since $P$ is pseudosymmetric, the definition of $h(y)$ does not depend upon the point $x$. Now suppose that $h\left(y_{1}\right)=h\left(y_{2}\right)$. Then $P\left(-x_{1}\right)=P\left(-x_{2}\right)$ where $P\left(x_{1}\right)=y_{1}$ and $P\left(x_{2}\right)=$ $y_{2}$. But $P\left(-x_{1}\right)=P\left(-x_{2}\right)$ implies $P\left(x_{1}\right)=P\left(x_{2}\right)$ again because $P$ is pseudosymmetric. Thus $y_{1}=y_{2}$ and $h$ is injective. In addition, it is surjective since $P$ is surjective and it follows immediately from (2.3.1) that

$$
h \circ P(x)=P(-x) \text { for each } x \in R \text {. }
$$

Among other things, this means that $h \circ P$ is continuous so that for any closed subset $H$ of $R$ we have $(h \circ P)^{-1}[H]=P^{-1}\left[h^{-1}[H]\right]$ is also closed and since any polynomial is a quotient map, this means $h^{-1}[H]$ is closed. Thus $h$ is continuous and since it is a bijection from $R$ onto $R$, this means that $h$ is a homeomorphism. Now define a mapping $t$ from $R$ onto $R$ by $t(x)=-x$. It then follows from (2.3.2) that

$$
h \circ P=P \circ t \text {. }
$$

We define a mapping $\varphi$ from $N_{P}$ into itself by $\varphi(f)=t \circ f \circ h^{-1}$. Since both $t$ and $h$ are homeomorphisms, $\varphi$ is a bijection and one easily shows that it is additive. To see 
that it is also multiplicative, use (2.3.3) and get

$$
\begin{aligned}
\varphi(f) \varphi(g) & =t \circ f \circ h^{-1} \circ P \circ t \circ g \circ h^{-1}=t \circ f \circ h^{-1} \circ h \circ P \circ g \circ h^{-1} \\
& =t \circ f \circ P \circ g \circ h^{-1}=t \circ f g \circ h^{-1}=\varphi(f g) .
\end{aligned}
$$

Thus, $\varphi$ is an automorphism of $N_{P}$.

We want to show now that $\varphi$ is not the identity automorphism. Suppose it is and for any real number $x$, let $\langle x\rangle$ denote the constant function which maps everything into $x$. Then

$$
\langle x\rangle=\varphi\langle x\rangle=t \circ\langle x\rangle \circ h^{-1}=\langle t(x)\rangle .
$$

This implies $t(x)=x$ for all $x$ which is a contradiction. Consequently, $\varphi$ is not the identity automorphism and the sufficiency portion of the proof has been established.

Now suppose Aut $N_{P}$ is isomorphic to $Z_{2}$. Then $P$ is not a homeomorphism in view of Theorem (2.1). Let $\varphi$ be the automorphism which is different from the identity. Then by Theorem (3.1) of $(2$, p. 286) there exists a homeomorphism $h$ from $R$ onto $R$ and a topological isomorphism $t$ from the additive group $R$ of real numbers such that for each $f \in N_{P}$ the diagram (2.1.5) commutes. Just as in the proof of Theorem 2.1, there is a nonzero real number $a$ such that $t(x)=a x$ for all $x \in R$ and if $a \neq 1,-1$, then $P$ is a homeomorphism which is a contradiction. Suppose $a=1$, that is, $t$ is the identity map. Then, according to diagram (2.1.5), $h(P(x))=P(t(x))=P(x)$ for all $x$ which, since $P$ is surjective, implies that $h$ also is the identity map. This, in turn, implies that $\varphi$ is the identity automorphism which is a contradiction. Consequently, $a=-1$ and we have $t(x)=-x$ for all $x \in R$. It then follows from diagram (2.1.5) that if $P(x)=P(y)$ then

$$
P(-x)=P(t(x))=h(P(x))=h(P(y))=P(t(y))=P(-y) .
$$

In other words, $P$ is pseudosymmetric and the proof is complete.

The following corollary is an immediate consequence of the previous two theorems.

Corollary 2.4. The near-ring $N_{P}$ is rigid (that is, admits only the identity automorphism) if and only if $P$ is not pseudosymmetric.

\section{Discussion, examples and further results}

In view of the crucial role played by pseudosymmetric polynomials in these considerations, it is appropriate to discuss them in some more detail. When we speak of a symmetric function here we mean a continuous selfmap of $R$ whose graph is symmetric about the origin, in other words, a continuous function $f$ such that $f(-x)=-f(x)$ for each $x \in R$. Of course, the symmetric polynomials are simply those polynomials with no terms of even degree. Evidently, every symmetric polynomial is pseudosymmetric. To get a pseudosymmetric polynomial which is not symmetric, one can take any symmetric polynomial and add to it any nonzero constant. There are less trivial examples and the following result whose easy verification is omitted gives a recipe for constructing them. 
Proposition 3.1. Let $Q$ be a symmetric polynomial and let $T$ be any polynomial which is a homeomorphism from $R$ onto $R$. Then $T \circ Q$ is pseudosymmetric.

Example 3.2. Take $Q(x)=x^{3}-x$ and $T(x)=x^{3}+x^{2}+x$. Then

$$
P(x)=T(Q(x))=x^{9}-3 x^{7}+x^{6}+3 x^{5}-2 x^{4}+x^{2}-x
$$

which is certainly not symmetric but according to the previous result is pseudosymmetric. It is immediate from Theorem 2.3 that for this particular polynomial $P$, Aut $N_{P}$ is isomorphic to $Z_{2}$.

As we observed previously, an odd degree polynomial with no even degree terms save the constant term is pseudosymmetric. In view of Example 3.2 the converse is not true. In the next result we give additional conditions which will force the converse.

Proposition 3.3. Let $P$ be any odd degree polynomial such that the zeros of its derived polynomial are all real and distinct. Then $P$ is pseudosymmetric if and only if it has no terms of even degree with the possible exception of the constant term.

It is convenient to have the following

Lemma 3.4. Suppose $P$ is a pseudosymmetric polynomial and has a local maximum or a local minimum at the point a. Then it also has a local maximum or a local minimum at the point $-a$.

Proof. Choose an increasing sequence $\left\{x_{n}\right\}$ and a decreasing sequence $\left\{y_{n}\right\}$ both converging to $a$ and satisfying $P\left(x_{n}\right)=P\left(y_{n}\right)$ for each $n$. Since the polynomial $P$ is pseudosymmetric, it takes on either a maximum value in the interior of $\left[-y_{n},-x_{n}\right]$ or a minimum value there for each $n$. For sufficiently large $n$, this must happen at the same point and that point can only be $-a$. Consequently, $P$ has either a local maximum or a local minimum at $-a$.

Now we are in a position to prove Proposition 3.3. Sufficiency is immediate and we say no more about it. As for necessity, let $\left\{a_{i}\right\}_{i=1}^{N}$ denote the positive real numbers at which $P$ has either a local maximum or a local minimum. Then according to Lemma 3.4, $P$ also has local maxima and minima at the points $\left\{-a_{i}\right\}_{i=1}^{N}$. Now $P$ cannot have a local maximum or a local minimum at 0 since this would mean that $P$ has an odd number of local maxima and local minima and this would force the degree of $P$ to be even. It readily follows

$$
P^{\prime}(x)=b\left(x-a_{1}\right) \ldots\left(x-a_{N}\right)\left(x+a_{1}\right) \ldots\left(x+a_{N}\right)=b\left(x^{2}-a_{1}^{2}\right) \ldots\left(x^{2}-a_{N}^{2}\right) .
$$

Thus $\boldsymbol{P}^{\prime}$ has only terms of even degree and it readily follows that $\boldsymbol{P}$ has no even degree terms except for possibly a nonzero constant term.

For a cubic polynomial $P$, one can determine the automorphism group of the near-ring $N_{P}$ simply by inspecting the coefficients of the polynomial. The relevant information is contained in the following. 


\section{Then}

Proposition 3.5. Let $P(x)=a x^{3}+b x^{2}+c x+d(a \neq 0)$ be any cubic polynomial.

Aut $N_{P}$ is isomorphic to $R_{M}$ the multiplicative group of nonzero real numbers if and only if $b^{2} \leqslant 3 a c$.

Aut $N_{P}$ is isomorphic to $Z_{2}$ if and only if $b=0$ and $c a<0$.

Aut $N_{P}$ consists of the identity automorphism if and only if $b \neq 0$ and $3 a c<b^{2}$.

Statement (3.5.1) follows from Theorem 2.1. Statement (3.5.2) follows from Theorem 2.3 and Proposition 3.3 and statement (3.5.3) follows from Corollary 2.4 and Proposition 3.3.

\section{REFERENCES}

(1) K. D. Magill, JR., Semigroup structures for families of functions, II; continuous functions, J. Aust. Math. Soc. 7 (1967), 95-107.

(2) K. D. MAGILL, JR., Semigroups and near-rings of continuous functions, General Topology and its Relations to Modern Analysis and Algebra, III, Proc. Third Prague Top. Symp., 1971 (Academia, Prague, 1972), 283-288.

State University of New York at Buffalo 